\title{
An Alternative Approach to Combine Orbital Prosthesis and Obturator: A Case Report
}

\author{
Gokce Sogancia \\ Suat Yalugb \\ Mustafa Kocaciklic
}

\section{ABSTRACT}

This report describes a different approach for diminishing the movements of orbital prosthesis during mimic movements and chewing function. Mechanical devices such as magnets are used to enhance the retention in case lack of the implants. However rigid fixation of obturator and orbital prosthesis can result in movements of the orbital prosthesis during mastication. In this case obturator and orbital prosthesis are combined by magnets. However this combination is not rigid because of an active part which provides movement. This active part allows movements in a space constructed in the acrylic base of orbital prosthesis. Thus, the movements that may occur on orbital prosthesis during chewing can be diminished by this simple mechanism. Due to the fact that designing such a mechanism that minimizes the movements of the orbital prosthesis contributes patient's comfort. (Eur J Dent 2011;5:459-464)

Key words: Combined prostheses; Magnets; Movement; Obturator; Orbital prosthesis.

\section{INTRODUCTION}

Radical maxillectomy frequently leads to extended defects in hard and soft tissues that result in a connection between the oral and nasal cavities. ${ }^{1-3}$ Maxillary defects, such as those created after extended tumor ablation, trauma or created

a Research Assistant, Gazi University, Faculty of Dentistry, Department of Prosthodontics, Ankara, Turkey.

b Professor, Gazi University, Faculty of Dentistry, Department of Prosthodontics, Ankara, Turkey.

c Lecturer, Gazi University, Faculty of Dentistry, Department of Prosthodontics, Ankara, Turkey.

- Corresponding author: Gokce Soganci Department of Prosthodontics, Faculty of Dentistry, Gazi University, 8.Cd. 82.Sk. No:4 Emek, 06510, Ankara, Turkey.

Phone: + 905326420486

Fax: + 903122239226

E-mail: dtgokcesoganciayahoo.com by congenital malformation, ${ }^{4}$ may result in severe facial disfigurement and compromised function. ${ }^{5}$ Lack of support, retention, and stability are common prosthodontic treatment problems for patients who have had a maxillectomy. 4,6 And also patients experience a loss of quality of life and feel isolated because of their appearance and functional deficits. ${ }^{5,7}$ Factors that affect the prosthetic prognosis for these patients are the size of defect, number of remaining teeth ${ }^{6,8}$ amount of remaining bony structure, quality of existing mucosa, radiation therapy, and patient's own ability to adapt to the prosthesis. Patients, who have unilateral resection with few remaining teeth, have problems like poor prosthetic prognosis because of inadequate denture bearing area, lack of cross arch stabilization, and lack of structures for denture 
retention ${ }^{6}$ as in completely edentulous patients. Oral rehabilitation after hemimaxillectomy presents diverse clinical and technical problems. ${ }^{9}$

A closed, hollow obturator is frequently used to restore anatomic form after radical surgery for maxillary cancer. ${ }^{10}$ Patients who have hollow obturators may complain of food, fluid, and mucous accumulations that result in bad odors and altered taste sensation. ${ }^{11}$ The closed hollow obturator prosthesis can prevent fluid and food collection, reduce air space, and allow for maximum extension. ${ }^{12}$ Using a technique for quick conversion of an obturator into a hollow bulb gives advantages. Therefore, the prosthesis is simple to construct, lightweight, and easy to clean; it has no direct junction between the oral, nasal, or antral environments and the interior of the obturator. ${ }^{11}$

Maxillofacial prosthetics is a recognized subspecialty of prosthodontics. ${ }^{13}$ Fabrication of the facial prostheses depends on experience of the prosthodontist. A key factor of a successful facial prosthesis is the extent and location of the defect, ${ }^{14}$ the retention of the prosthesis to the patients skin, and retention of a facial prosthesis with no mechanical means depends primarily on medical skin adhesives, ${ }^{15}$ adhesive tapes and undercuts. ${ }^{16}$ The use of magnets is the most efficient means of providing combined prostheses with retention and stability in patients with deformities requiring complex rehabilitations. The majority of prostheses with magnets are sectioned and have a magnet in each section. ${ }^{2}$ Bone anchored implants are also used to retain extraoral prostheses. ${ }^{17} \mathrm{Fa}-$ cial implants have advantage of being able to attach the prosthesis firmly to the patients face. ${ }^{16}$ However an important factor that must be taken into account when considering implant retention for facial prostheses is the economical situation of the patient to afford implants.

It is essential to incorporate both the orbital prosthesis and obturator properly. This article describes a procedure in a case for diminishing the movements of orbital prosthesis that can occur due to mimic and chewing functions.

\section{CASE REPORT}

A 46 year-old male patient had a complaint of his facial esthetic and loss of masticatory function and speech. Depends on information obtained from the patient, he had an operation due to a mixed tumor of parotid gland in the hospital of Gazi University in 2001. Then radiotherapy was applied. 2 years after the removal of the left maxilla, he had a second operation from his left orbital region because of metastasis. After healing period, he scheduled to Gazi University Faculty of Dentistry in order to make his obturator and orbital prosthesis (Figure 1a, b). During the prosthodontic visit remaining structures and the surgical defect was examined. The clinical findings indicated that the patient had a canine and lateral teeth with combined crowns in the maxilla. Resection site included the half of the maxilla which was classified as Aramany class I. Surgical procedure resulted with an opening between oral, nasal and orbital cavities. Lateral incisor had to be extracted because of luxation. After healing period, porcelain crown of the canine was renewed. Due to the lack of sufficient structures and undercuts for retention of orbital prosthesis, as well as obturator, it was planned to combine both of the prostheses. Implant was recommended to the patient, however he did not accept the implant treatment. In this case combination of the orbital prosthesis and obturator accomplished with an alternative method. It was aimed to reduce dislodgement of the orbital prosthesis combined with obturator by means of an active part during mastication.

Primarily, a conventional hollowed obturator partial denture prosthesis was fabricated in the usual manner. ${ }^{16}$ Existing hollow obturator was converted into a hollow bulb by a simple and quick technique ${ }^{11}$ using an autopolymerizing acrylic resin (Kemdent, Associated Dental Products Ltd, Wiltshire, UK). Necessary corrections were made after the prosthesis has been worn for a few days.

After finishing the obturator, an impression of the orbital defect was made with irreversible hydrocolloid impression material (Tulip, Cavex, Haarlem, Holland). Type 3 hard dental stone (Labstone, Heraeus Kulzer, Armonk, NY) was poured into the impression to get the definitive cast for sculpting. An acrylic resin (Akribel, Atlas-Enta AS., Izmir, Turkeyl core was fabricated on the cast for attaching the ocular part of the orbital prosthesis. This acrylic core was acted as a base both for attaching the ocular part and for constructing a simple mechanism that resembles a suspension system between obturator and orbital prosthesis. As it is seen in Figure 2, the ocular part was ad- 
justed according to the symmetrical eye and the wax pattern of the orbital prosthesis was then fabricated. After try in stage, the wax model was invested into the mold, and the orbital prosthesis was fabricated from silicone (Cosmesil, Principality Medical Limited, Newport-South Wales, UK) using conventional techniques. ${ }^{17}$

Cobalt samarium magnets, illustrated in Figure 3, (Manyet, Manyetik Tutucular Ltd, Istanbul, Turkeyl were used to combine the obturator to orbital prosthesis with an active mechanism. Initially, a hole was opened on the closed hollow bulb to locate the magnet. Magnets were roughened with a diamond bur to enhance the retention of acrylic resin. Autopolymerizing acrylic resin (Kemdent, Associated Dental Products Ltd, Wiltshire, UKJ was prepared and placed the hole together with the magnet. Excess amount of acrylic resin was removed and bulb was polished after polymerization was completed. Subsequently, a

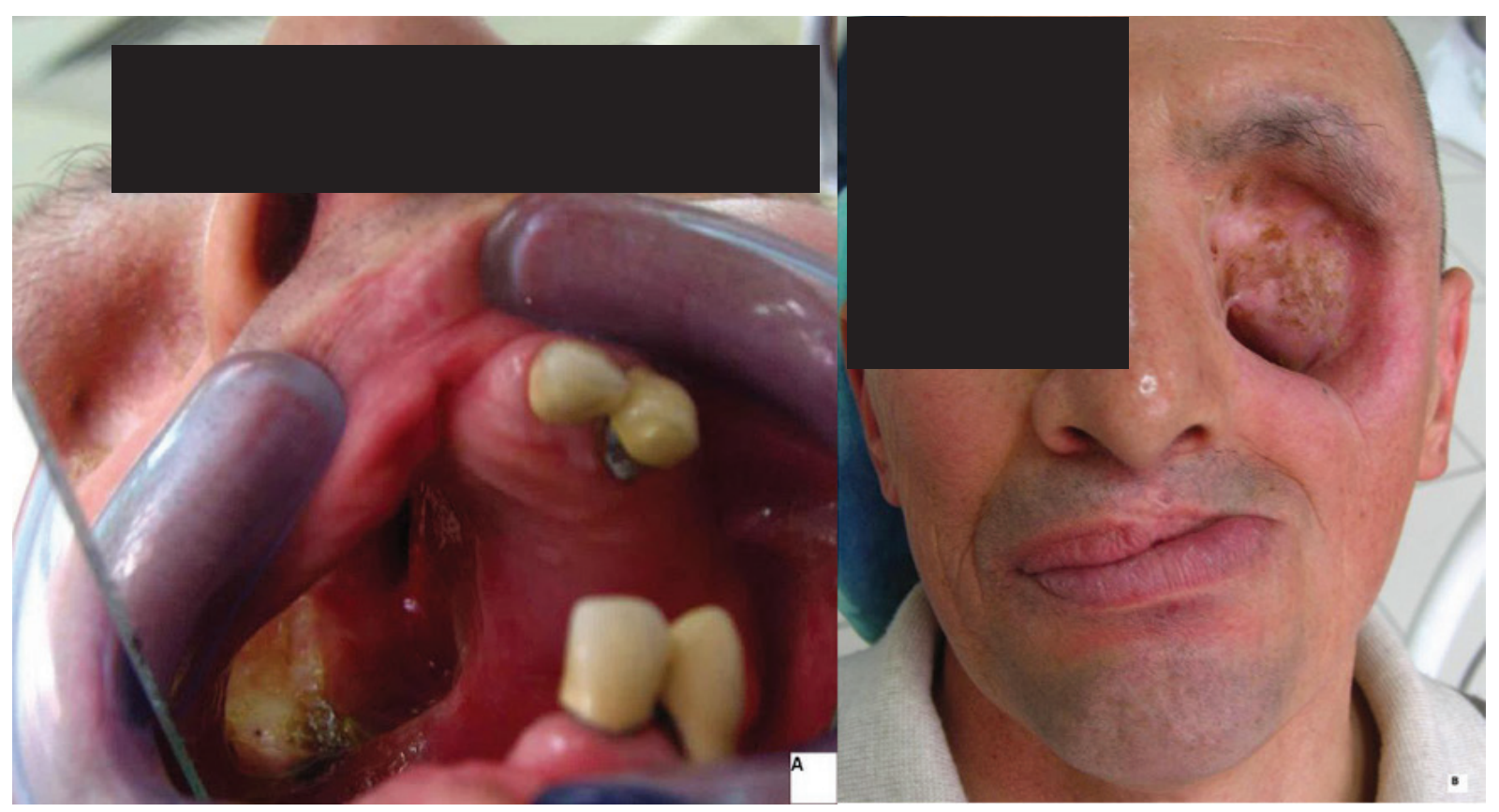

Figure 1. A. Intra-oral view before treatment. B. Extra-oral view of the orbital defect.

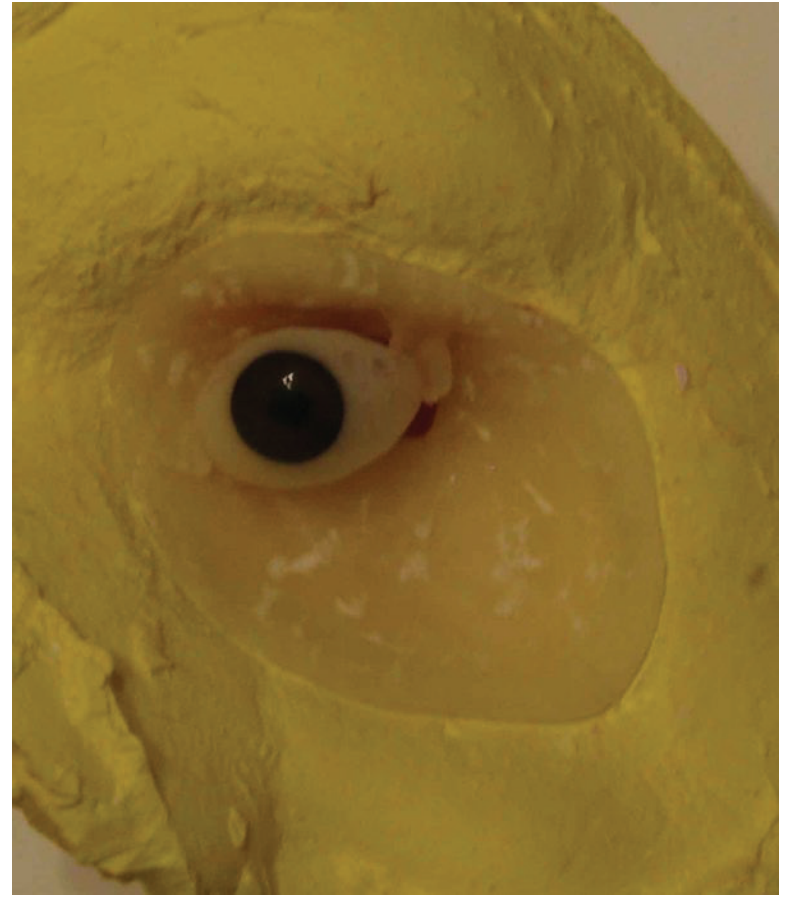

Figure 2. Acrylic resin core with ocular part adjusted according to the symmetrical eye.

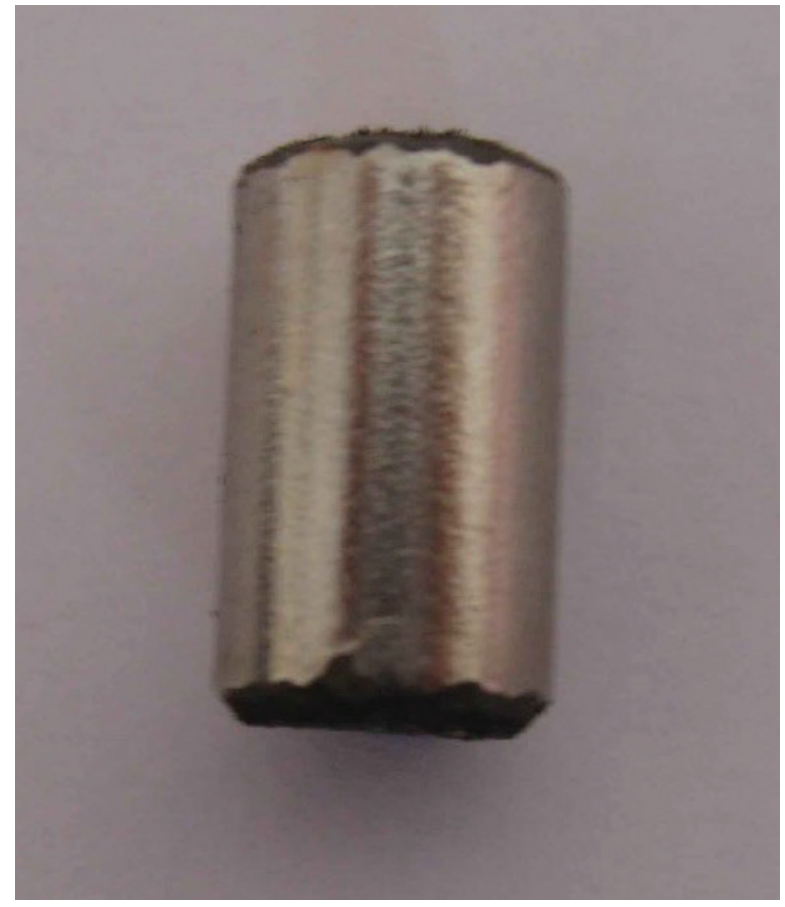

Figure 3. Cobalt samarium magnet used to combine the prostheses. 


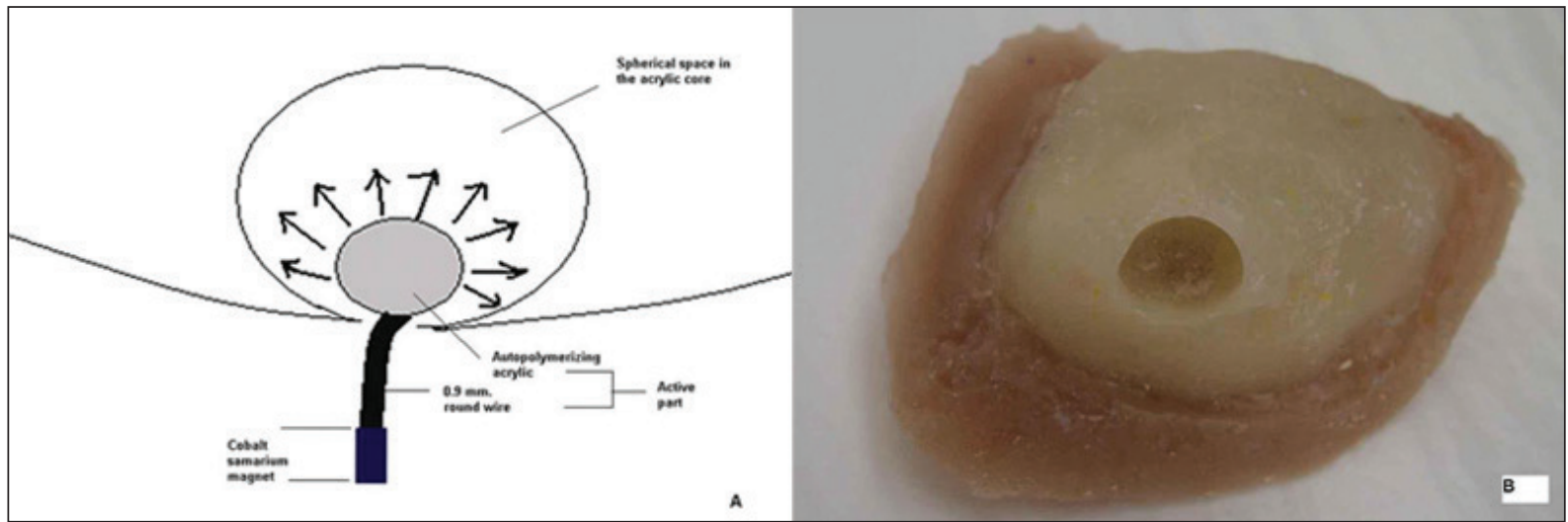

Figure 4. A. Schematic view of active part and spherical hole in the acrylic resin core. The active part can move in all directions inside this hole during chewing. B. Spherical hole prepared in the acrylic resin core adjacent to the oral cavity.

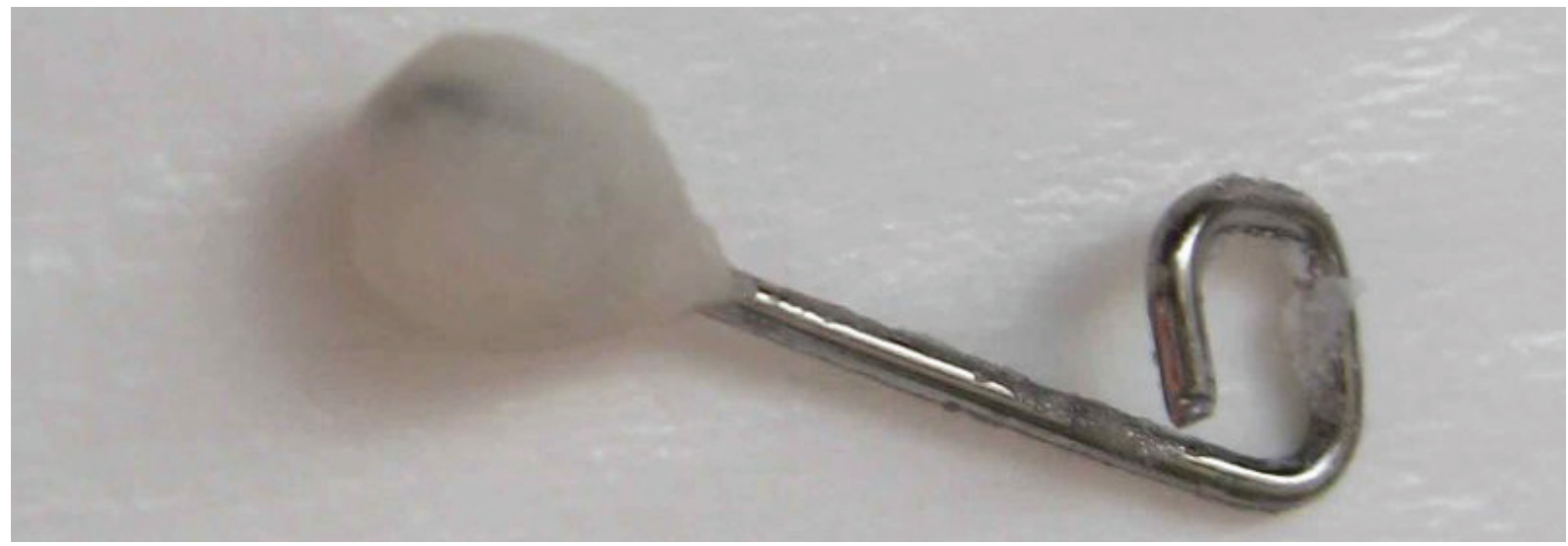

Figure 5. Active part with round shaped acrylic resin on the tip.

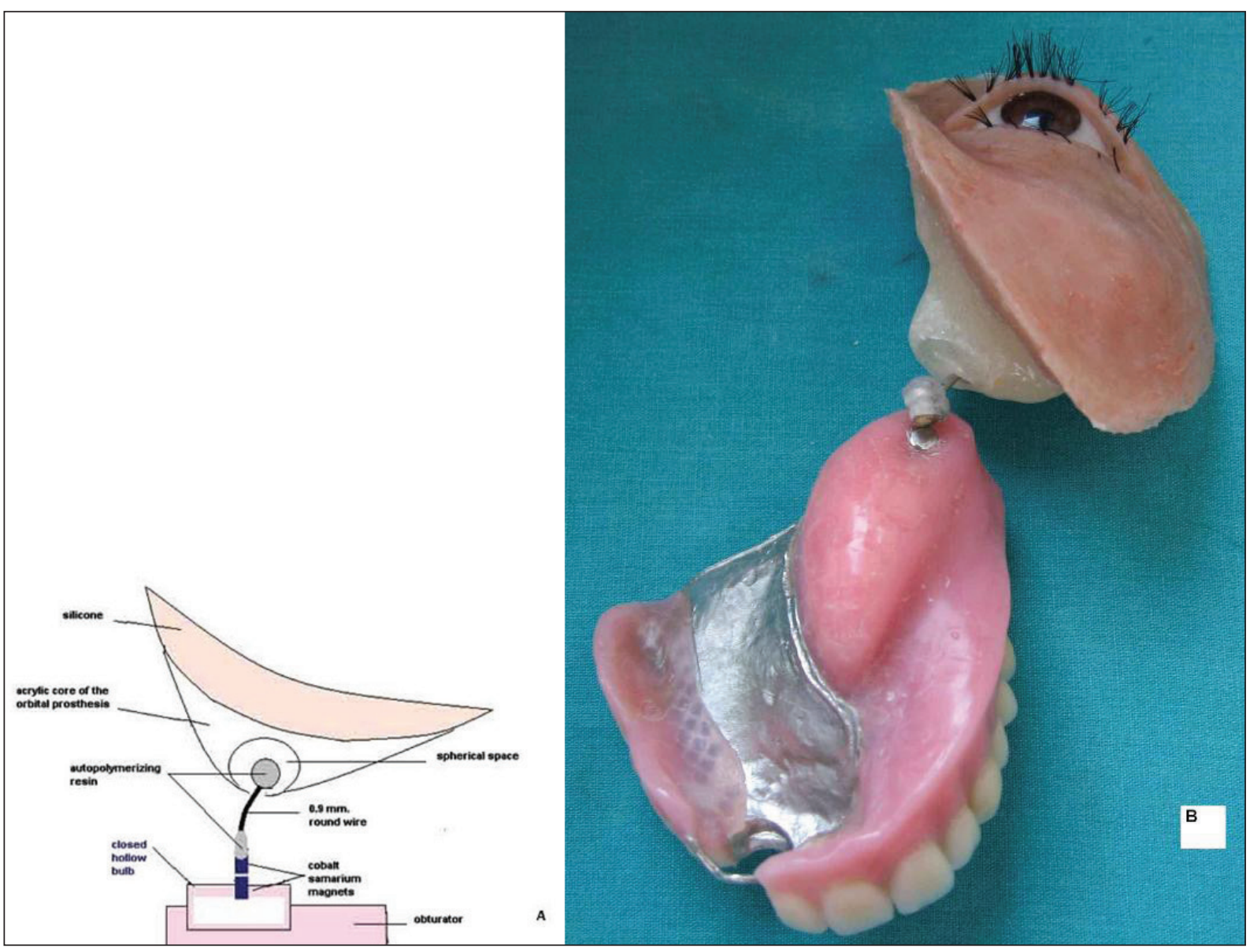

Figure 6. A. Schematic view of combined prostheses. B. Obturator and orbital prosthesis combined with magnets by means of active part. 
spherical large hole that function as a space for suspension system in the core of the orbital prosthesis were prepared. This hole was drilled using steel round bur (Medin, Joint Stock Company, Vlachovicka, Czech Republic) to make the spherical space in the part of acrylic core which is adjacent to the oral cavity. The thought whilst making the hole in spherical shape was to have the movement of active part provided in vertical, horizontal and oblique directions in this hole (Figure $4 a, b$ ).

In order to make this active part, a $0.9 \mathrm{~mm}$ round wire (Dentaurum, The Dentaurum Group, Pforzheim, Germanyl was taken, and cut in measured length between closed hollow bulb and buttom surface of acrylic core. The tip of the wire was curved due to contribute the autopolymerized acrylic resin to retain. A spherical shape was given to acrylic resin on the curved tip of wire correspond to the large spherical hole prepared before in the acrylic core of orbital prosthesis (Figure 5). Other tip of the wire was attached to the magnet also using autopolymerizing acrylic resin.

Tip of the active part with round shaped acrylic resin was inserted into the spherical hole in acrylic core. Autopolymerizing acrylic resin was added to the border of the hole to reduce the opening due to keep the active part in. Thus, one tip of the active part would be in the hole to provide the movement in vertical, horizontal and oblique directions during mastication and the other tip with magnet would ensure the connection between the obturator and the orbital prosthesis (Figure 6a, b).

Prostheses were tried in place and magnets were controlled if they function properly (Figure 7). Necessary controls were done to confirm keeping the movement of the orbital prosthesis in minimal bounds whilst the patient makes chewing functions and mimic movements.

\section{DISCUSSION}

This article describes a different procedure for diminishing the movement between orbital prosthesis and obturator connecting with magnets by means of an active part.

Adhesive systems and spectacle frame are used when orbital prosthesis cannot be retained by osseointegrated implants. With few or missing undercuts, however, mimic motion and sneezing can cause adhesive failure. Thus, the orbital prosthesis may be attached to the obturator with mag- nets or buttons. ${ }^{1}$ The use of magnets is the most efficient means of providing combined prostheses with retention and stability in patients with deformities requiring complex rehabilitations. ${ }^{2}$ Movement of the orbital prosthesis can occur during chewing in case the connection with obturator is rigid. In a case report proclaimed by Goiata et al, ${ }^{2}$ connection between orbital prosthesis and obturator were procured directly by magnets. Due to the excess amount of maxillary bone loss, obturator can lose the stability and move vertically. ${ }^{1}$ In such cases, movement of orbital prosthesis away from the tissue can occur if the connection is rigid between the prostheses. The prostheses which are rigidly attached by magnets can have retention failure during chewing functions because of this masticatory strain. ${ }^{1}$ Thus, it is resulted in loss of contact of the silicone prosthesis margins. For that reason, thought to have the benefit of more stable prostheses in this case was to construct an active mechanism which compansates the movement during function. Wieselmann-Penker et $\mathrm{al}^{1}$ fabricated a spring-loaded rewinding device for orbital prosthesis to stay in situ during mastication and various mimic movements. With the similar idea the active mechanism is thought in this case to prevent the movement of orbital prosthesis. A large spherical hole was prepared in the base of the orbital prosthesis on account of the

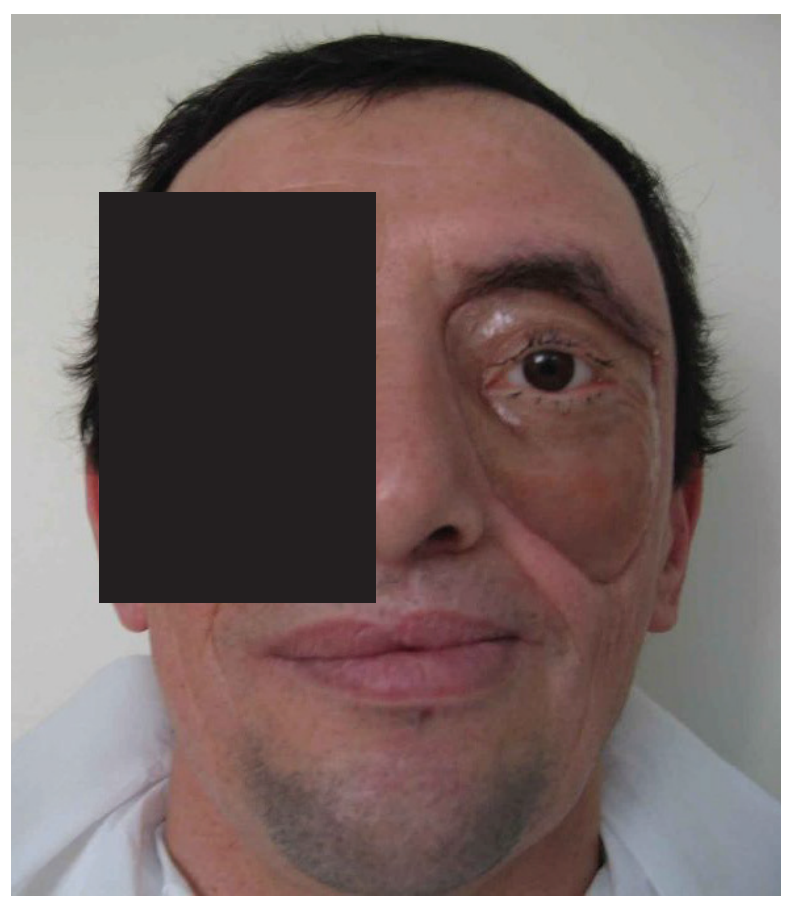

Figure 7. View of the orbital prosthesis and lip support whilst the obturator is in situ after treatment. 
fact that there is a need for compansating vertical movement of the obturator during chewing. Obturator with minimum tooth and tissue support can move vertically, horizontally or oblique directions at the time of chewing. To prevent the movement of orbital prosthesis together with obturator, there should be a space to compensate this movement. Whilst the patient chews, active part will move in the spherical hole so that obturator cannot push the orbital prosthesis away from its place. This spherical hole act as a suspension mechanism. The reason for constructing this hole in spherical shape is directions of movement. During chewing not only vertical movements take place but also oblique and horizontal movements can be seen. In order to correspond these directions the hole was constructed in spherical shape.

In spite of providing the orbital prosthesis to stay in situ during mastication, the active mechanism also has a function to pull prostheses to each other via magnets during rest times.

In order to enhance the retention of both orbital prosthesis and obturator, this technique can be an alternative method instead of using adhesives in such cases that endoosseos implants cannot be used.

\section{REFERENCES}

1. Wieselmann-Penkner K, Arnetzl G, Mayer W, Bratschko R. Minimizing movement of an orbital prosthesis retained by an obturator prosthesis. J Prosthet Dent 2004;91:188-190.

2. Goiato MC, Fernandes AU, dos Santos DM, Barão VA. Positioning magnets on a multiple/sectional maxillofacial prosthesis. J Contemp Dent Pract 2007;8:101-107.

3. Koyama S, Sasaki K, Inai T, Watanabe M. Effects of defect configuration, size, and remaining teeth on masticatory function in post-maxillectomy patients. J Oral Rehabil 2005;32:635-641.

4. Keyf F. Obturator prostheses for hemimaxillectomy patients. J Oral Rehabil 2001;28:821-829.

5. Depprich RA, Handschel JG, Meyer U, Meissner G. Comparison of prevalence of microorganisms on titanium and silicone/polymethyl methacrylate obturators used for rehabilitation of maxillary defects. J Prosthet Dent 2008;99:400-405.

6. Wang RR. Sectional prosthesis for total maxillectomy patients: A clinical report. J Prosthet Dent 1997;78:241-244.
7. Goiato MC, Pesqueira AA, Ramos da Silva C, Filho HG, Micheline dos Santos D. Patient satisfaction with maxillofacial prosthesis. Literature review. J Plast Reconstr Aesthet Surg 2009;62:175-180.

8. Oki M, lida T, Mukohyama H, Tomizuka K, Takato T, Taniguchi $\mathrm{H}$. The vibratory characteristics of obturators with different bulb height and form designs. J Oral Rehabil 2006;33:43-51.

9. Rilo B, Dasilva JL, Ferros I, Mora MJ, Santana U. Hollowbulb interim obturator for maxillary resection: A case report. J Oral Rehabil 2005;32:234-236.

10. Nishigawa G, Maruo Y, Jin K, Oki K, Minagi S. A custommade sealing screw cap for a closed, hollow obturator. $J$ Craniofac Surg 2007;18:1138-1141.

11. Asher ES, Psillakis JJ, Piro JD, Wright RF. Technique for quick conversion of an obturator into a hollow bulb. J Prosthet Dent 2001;85:419-420.

12. Habib BH, Driscoll CF. Fabrication of a closed hollow obturator. J Prosthet Dent 2004;91:383-385.

13. Wolfaardt JF. Maxillofacial prosthetics--an international perspective of the British status quo. J Oral Rehabil 1992;19:1-11.

14. Kiat-amnuay S, Lemon JC, Wesley PJ. Technique for fabricating a lightweight, urethane-lined silicone orbital prosthesis. J Prosthet Dent 2001;86:210-213.

15. Kiat-amnuay S, Waters PJ, Roberts D, Gettleman L. Adhesive retention of silicone and chlorinated polyetylene for maxillofacial prosthesis. J Prosthet Dent 2008;99:483-488.

16. Seto K. Atlas of oral and maxillofacial rehabilitation. Tokyo: Quintessence Publishing Co., Ltd.; 2003; p.25-28.

17. McKinstry RE. Fundamentals of facial prosthetics. Arlington: ABI Professional Publications; 1995; p.121-136. 